\title{
Mechanism of grain refinement and its effect on Adiabatic Shear Bands in 4340 steel and pure copper during impact
}

\author{
Nabil Bassima and Solomon Boakye-Yiadom \\ Department of Mechanical Engineering, University of Manitoba, Winnipeg, MB, Canada, R3T5V6
}

\begin{abstract}
Pre-deformation and post-deformation microstructure characterization was conducted on tempered 4340 steel and commercial pure copper specimens under impact to determine the microstructural changes and the mechanism of grain refinement that occur during the evolution of ASBs. It was observed that the movement and multiplication of dislocations, elongation of grains, breaking of elongated grains, rotation, carbide fragmentation and boundary refinement of broken grains occur simultaneously during the evolution of ASBs in the impacted 4340 steel specimens. The extent of these mechanisms depends on the imposed local strain and strain rate. Extensive grain refinement coupled with high density of dislocations results in the shear band structures being more susceptible to crack nucleation and propagation. In copper, it was observed that sequential occurrence of emergence of dislocations, dislocation cell formations with varying cell boundaries and cell interiors, dynamic recovery and extensive micro-twinning results in the formation of the shear bands. The structure within the evolved shear bands becomes less brittle after the onset of dynamic recovery and micro-twinning. The differences in the mechanism of grain refinement and evolution of the shear bands in both materials is attributed to the differences in the mobility of dislocations, the rate of strain hardening and strain hardening exponents in both materials studied.
\end{abstract}

\section{Introduction}

Metals and other materials exhibit the formation of narrow bands of extreme strains known as Adiabatic Shear Bands (ASBs) when deformed at high strain rates and large strains [1]. Mechanical and material factors have been reported to influence the appearance and susceptibility of materials to the formation of ASBs [1]. It has been reported that $\mathrm{BCC}$ metals exhibit an increased propensity to ASB when their grain sizes are reduced into the ultrafine-grain and nano-crystalline regime [2]. This is attributed to the associated changes in the strength, strain hardening behaviour and strain rate sensitivity when the grain size changes [2]. Also, it has been observed that the ASBs associated with FCC metals, such as aluminium and copper, are diffused and exhibit a plastic flow phenomenon [3]. The influence of these factors on the formation of ASBs during dynamic loading makes the phenomenon of ASBs very complex.

In this study, pre-impact and post-impact microstructure characterization was conducted on tempered 4340 steel (BCC) and pure copper (FCC) specimens to determine the mechanism of evolution of ASBs. This was done in order to determine the effect crystal structure and microstructure on the mechanisms and appearance of ASBs in both materials.

\section{Experimental procedure}

Heat treatable AISI 4340 steel (C: $0.38-0.43 \%$, Mn 0.6$0.8 \%$, Si $0.15-0.35 \%$, Cr 0.7-0.9\%, Mo 0.2-0.3, Ni 1.65$2.00 \%$ ) and copper of commercial purity (99.9\% purity)

\footnotetext{
${ }^{a}$ Corresponding author: author@email.org
}

were used in this study. As received rod forms of both specimens were machined into a diameter of $9.5 \mathrm{~mm}$ and a length of $10.5 \mathrm{~mm}$. The machined steel specimens were heat treated using an austenitize-quench-and-temper procedure prior to impact. The machined specimens were impacted at high strain rates and large strains using the Direct Impact Hopkinson Pressure Bar (DIHPB) at different projectile velocities resulting in several impact momentums. During the impact, an AISI 4340 steel projectile $(18.67 \mathrm{~N}, 38 \mathrm{~mm}$ diameter by $127 \mathrm{~mm}$ length) with a hardness of $47 \mathrm{HRC}$ is fired by a gas gun, and strikes the specimen attached to a transmitter bar at a high impact velocity $[4,5]$.

Selected specimens of both materials were studied prior to and after impact using metallographic and electron microscopy techniques. The microstructural analyses were conducted using a ZIESS Axiovert 25 invertedreflected light microscope, JEOL 5900 Scanning Electron Microscope (SEM), a JEOL 2100F TEM/STEM and a JEOL 2010F TEM to document the structure and appearance of the ASBs after impact.

\section{Results}

\subsection{Microstructural evolution of shear Bands in steel during impact}

The tempered steel specimens prior to impact consisted of precipitated platelet and spherical carbides within ferrite matrix (tempered martensite structure) as shown in Fig. 1. The predominant platelet carbides were aligned on the lath boundaries (interlath carbides) and within the laths (intralath carbides). Details of the initial structure of

This is an Open Access article distributed under the terms of the Creative Commons Attribution License 4.0, which permits unrestricted use, distribution, and reproduction in any medium, provided the original work is properly cited. 


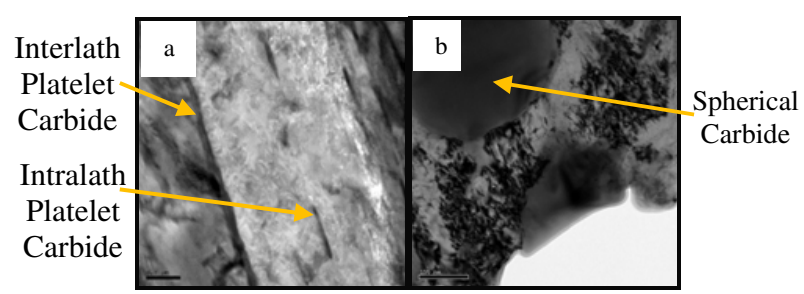

Figure 1. TEM bright field micrographs of the tempered steel specimens prior to impact.

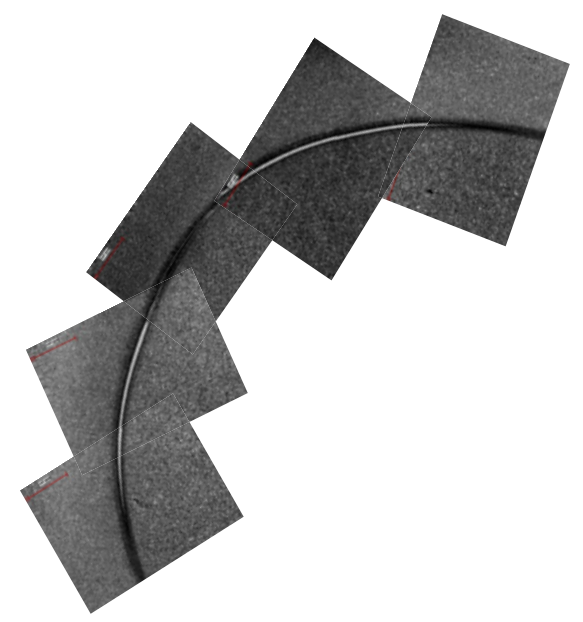

Figure 2. Optical micrographs of adiabatic shear band in a single steel specimen impacted at $51 \mathrm{~kg} \cdot \mathrm{m} / \mathrm{s}$.

the tempered steel specimens have been published in references $[4,5]$.

Figure 2 shows the trajectory of an evolved ASB in a single steel specimen impacted at $51 \mathrm{~kg} \cdot \mathrm{m} / \mathrm{s}$. The evolved shear bands were very hard and brittle compared to the regions outside the shear bands. This creates a hardness gradient across the microstructure of the impacted material. The structure of the shear bands become metallographically distinct as the impact momentum (strain rate) is increased.

At higher impact momentums (strain rates), cracks propagated along the lengths of the shear bands. Figure 3 shows cracks propagating along a shear band that evolved in a steel specimen after impact. The wavy nature of the propagating crack demonstrates void nucleation, growth and coalescence which develop into cracks along the length of the band. It is inferred that the connection between adjacent voids give the cracks their wavy nature prior to fracture and fragmentation as shown in Fig. 3.

Extensive microstructural analysis on the impacted steel specimens revealed that the evolved shear band microstructures were not uniform at any of the stages of the impact because several types of grains and structures were observed coexisting within the ASBs. At relatively lower impact momentum/strain rates, after the initiation of strain localization, the structure is comprised of mainly elongated grains and few broken grains within the ASB. After this stage, the structure that evolved after increasing the impact momentum/strain rate was made of predominantly broken grains; a few refined grains and nanograins, while yet another structure after increasing the strain

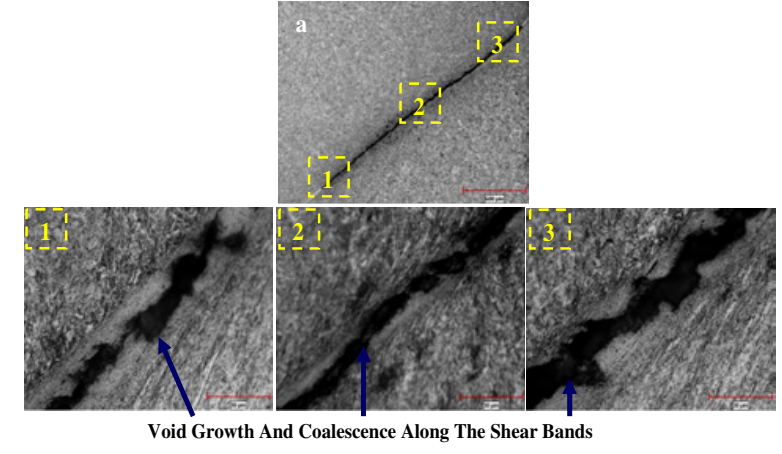

Figure 3. Optical micrographs of microvoids coalescing to form cracks along a shear band as shown in regions 1,2 and 3 .

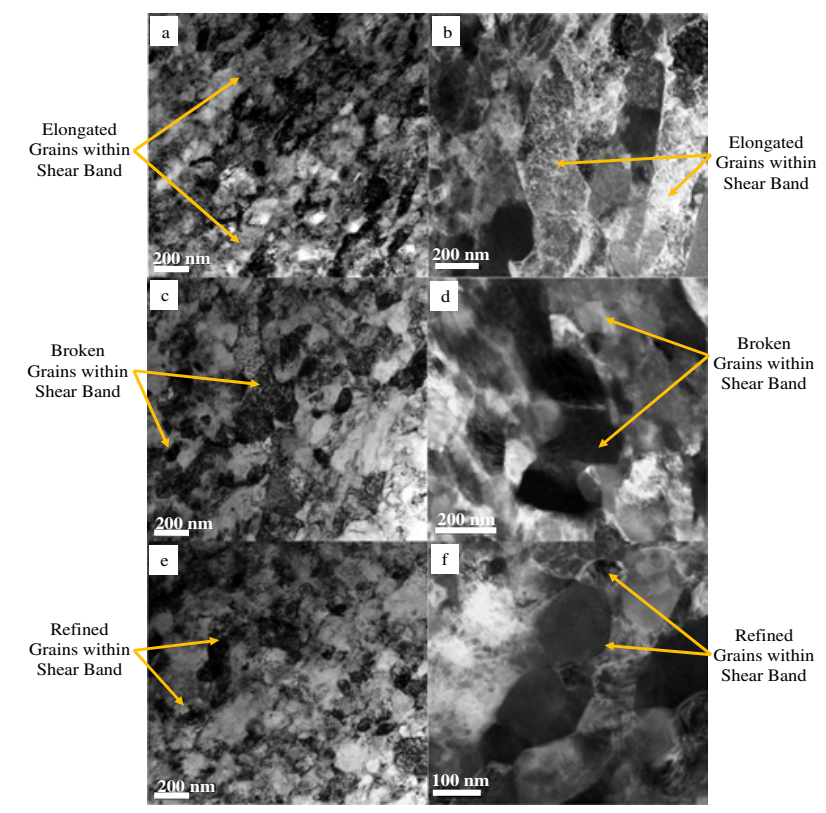

Figure 4. TEM micrographs showing (a), (b) elongated grains within shear band in the steel specimen impacted at $43 \mathrm{~kg} \cdot \mathrm{m} / \mathrm{s}$. (c), (d) broken grains within shear band in the steel specimen impacted at $47 \mathrm{~kg} \cdot \mathrm{m} / \mathrm{s}$. (e) (f) refined grains within shear bands in the steel specimen impacted at $50 \mathrm{~kg} \cdot \mathrm{m} / \mathrm{s}$.

rate was made of dominant refined grains, nanograins and subgrains. Detail description has been published in references 11 . and 12. Figure 4 shows TEM micrographs of the microstructures that evolved within the shear bands in the steel specimens after impact at 43,47 and $50 \mathrm{~kg} \cdot \mathrm{m} / \mathrm{s}$.

Figure 5 is a schematic of the stages of grain evolution that occurs within the evolving shear bands during impact of the steel specimens. It is based on the observations of the structure within ASBs as a function of strain rate/impact momentum. It is emphasized here that each of the stages represents an already failed material because of the presence of either a diffused or distinct ASB after impact. The occurrence of strain localization starts with texture development and the emergence of dislocations as shown in Fig. 5(a) and (b) which depicts the elongated grains with initiated transverse and random dislocation boundaries. At this stage, the elongated grains are decorated with high density of dislocations including clusters of dislocations along the predominant transverse dislocation 


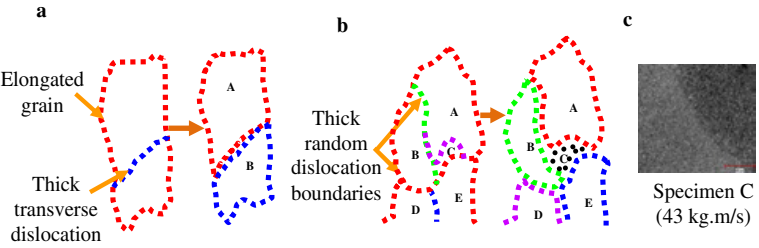

boundary
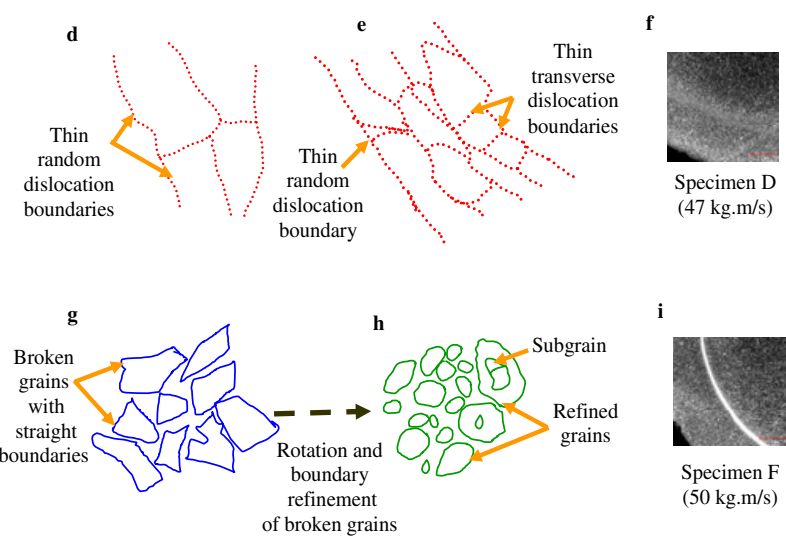

Figure 5. Schematic of the stages of grain refinement during the evolution of ASBs. (a), (b) Occurs after the initiation of strain localization which corresponds to the diffused ASB in (c). (d), (e) Occur prior to breaking of the elongated grains which corresponds to the ASB in (f). (g) Represents broken grains which undergo rotation and boundary refinement to produce refined grains (h) Refined grains and subgrains which corresponds to the ASB in (i).

boundaries. This represents a unique structure of ASB which corresponds to the observed metallographically diffused ASB shown in Fig. 5(c). Figure 5(d) and (e) illustrates a later stage after grain elongation prior to the breaking of the elongated grains along the initiated dislocation boundaries. The observed boundaries at this stage have thin clusters of dislocations along the initiated dislocation boundaries and correspond to the shear band in Fig. 5(f). The broken grains with smaller sizes and straight boundaries are shown in Fig. 5(g). At this stage, the observed dislocations were within the broken grains and not concentrated on the boundaries. Rotation and boundary refinement of the broken grains results in refined grains with round boundaries as shown in Fig. 5(h) which corresponds to the distinct ASB in Fig. 5(i).

\subsection{Microstructural evolution of shear bands in pure copper during impact}

Pre-impact microstructure of the pure copper specimens revealed that the structure is made of large grains and deformation twins as shown in Fig. 6. Dislocations were also observed both within the large grains and on the twins.

Figure 7(a) shows an optical micrograph of a copper specimen after impact at $45 \mathrm{~kg} \cdot \mathrm{m} / \mathrm{s}$. The shear bands were wide and appeared metallographically diffused from the surrounding regions. The thickness and appearance of the shear bands remained relatively the same (widediffused bands) regardless of the impact momentum/strain

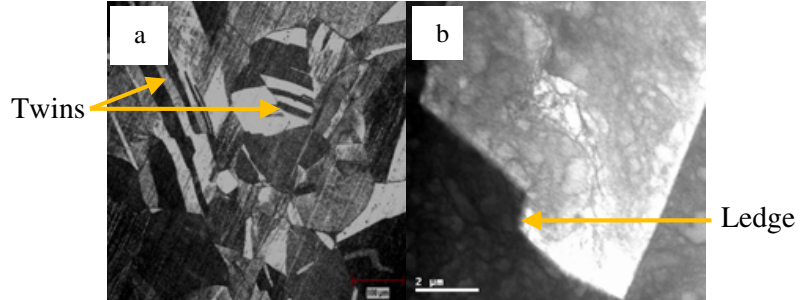

Figure 6. (a) Optical micrograph (b) TEM bright field micrograph of copper specimen prior to impact.

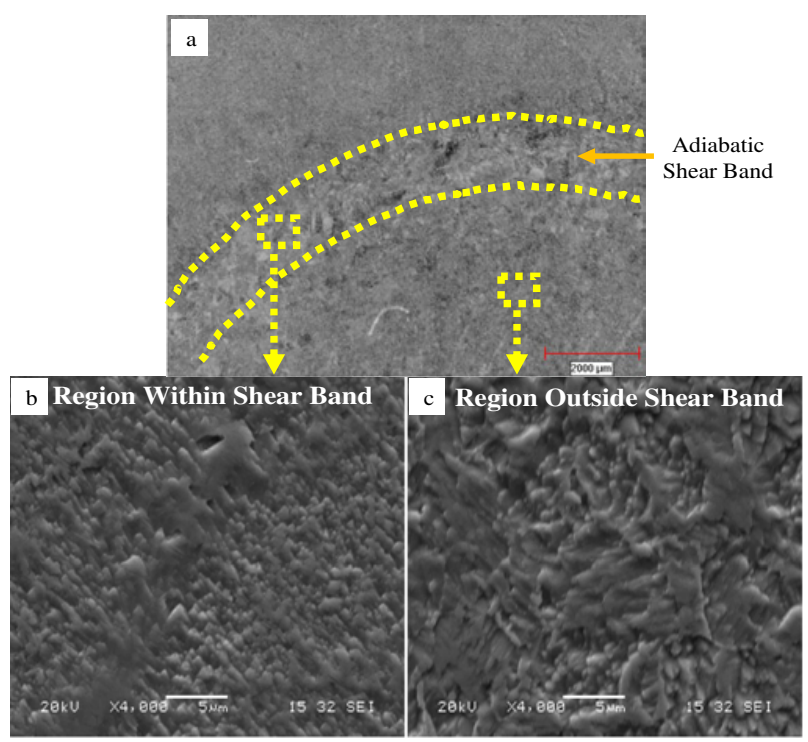

Figure 7. (a) Optical micrograph of ASB in a pure copper after impact at $45 \mathrm{~kg} \cdot \mathrm{m} / \mathrm{s} \mathrm{(b),} \mathrm{(c)} \mathrm{SEI} \mathrm{of} \mathrm{regions} \mathrm{within} \mathrm{and} \mathrm{outside}$ ASB respectively.

rate. This is in contrast to the shear bands observed in the impacted steel specimens. The shear bands in the steel specimens were wide and metallographically diffused at lower impact momentum/strain rates while they become thin and distinct from the surrounding regions as the impact momentum/strain rate increase. Figure 7(b) and (c) show Scanning Electron Images (SEI) of the shear band in Fig. 7(a). The effect of shear strain localization on the structure within the shear band compared to the structure outside the shear band becomes apparent (micrographs taken under the same conditions). The regions within the shear bands are highly strained compared to the regions outside the bands.

A comprehensive Transmission/Scanning Transmission Electron Microscopy (TEM/STEM) analysis on the impacted copper specimens revealed that there were extensive differences between the regions within the shear bands in the copper specimens impacted at relatively lower impact momentum/strain rates compared to the regions within the shear bands in the copper specimens impacted at high impact momentum/strain rates.

Figure 8(a) to (f) show the microstructure of the regions within the adiabatic shear bands that developed in the copper specimens impacted at $45 \mathrm{~kg} \cdot \mathrm{m} / \mathrm{s}$. Dislocation cell structures were the predominant features within the shear bands in this region. The dislocation cells had 


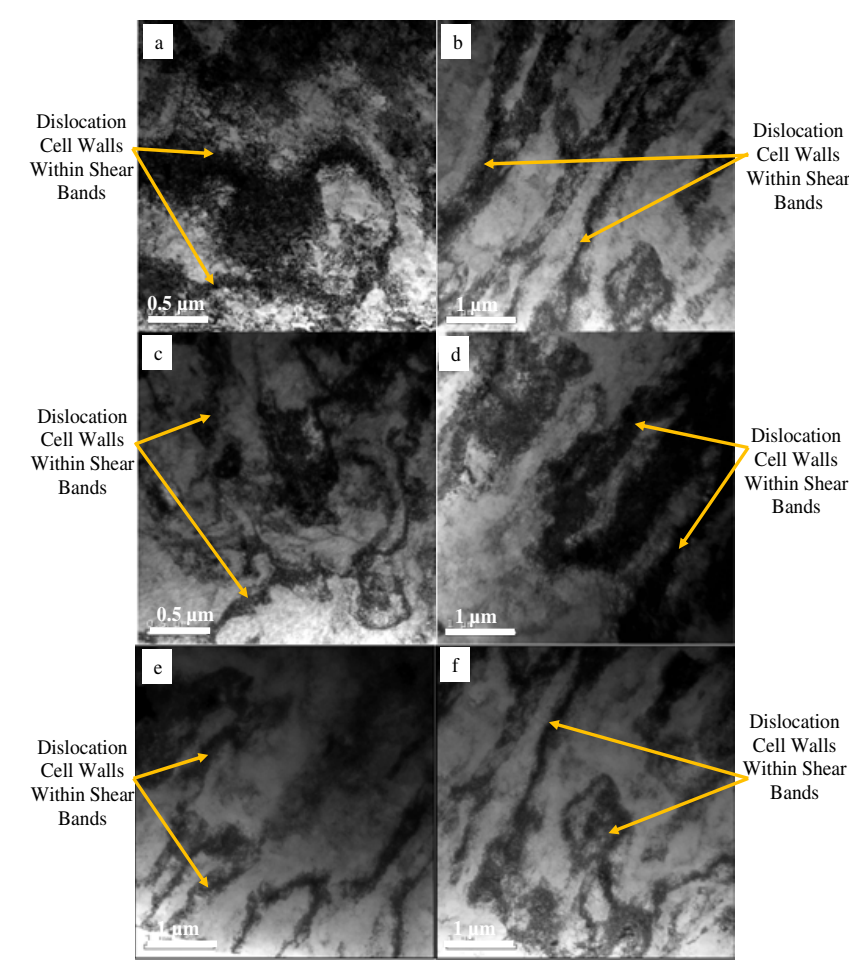

Figure 8. TEM micrographs of regions within an ASB that evolved in a copper specimen after impact at $45 \mathrm{~kg} \cdot \mathrm{m} / \mathrm{s}$ showing dislocation cell formation.

thick boundaries with clean cell interiors. Some of the dislocation cells had thicker cell boundaries with more dislocations within the cell interiors. It is inferred that the emergence of dislocations and the formation of dislocation cell boundaries are the predominant structures within adiabatic shear bands that evolve in copper at relatively lower strain rates as shown in Fig. 8.

At relatively higher strain rates, the adiabatic shear bands that evolved within the copper specimens were characterized by recrystallized grains and microtwins.

Figure 9 shows the microstructure of regions within evolved adiabatic shear bands in a copper specimen that was impacted at $57 \mathrm{~kg} \cdot \mathrm{m} / \mathrm{s}$. These recrystallized grains were well developed with distinct grain boundaries. The dislocation cells with predominantly thicker boundaries disappeared from the regions within the shear bands due to associated localized recovery and dynamic recrystallization mechanisms.

Annealing twins were also predominant within the shear bands. The annealing twins did not have dislocation structures within the grains compared to the observed dislocation structures within the deformation twins that were observed in the pre-impact copper specimens.

\section{Discussions}

The current study shows that the mechanism of evolution of adiabatic shear bands in tempered steel specimens are different from those observed in pure copper specimens. The onset of strain localization in the tempered steel specimens was characterized by the emergence of

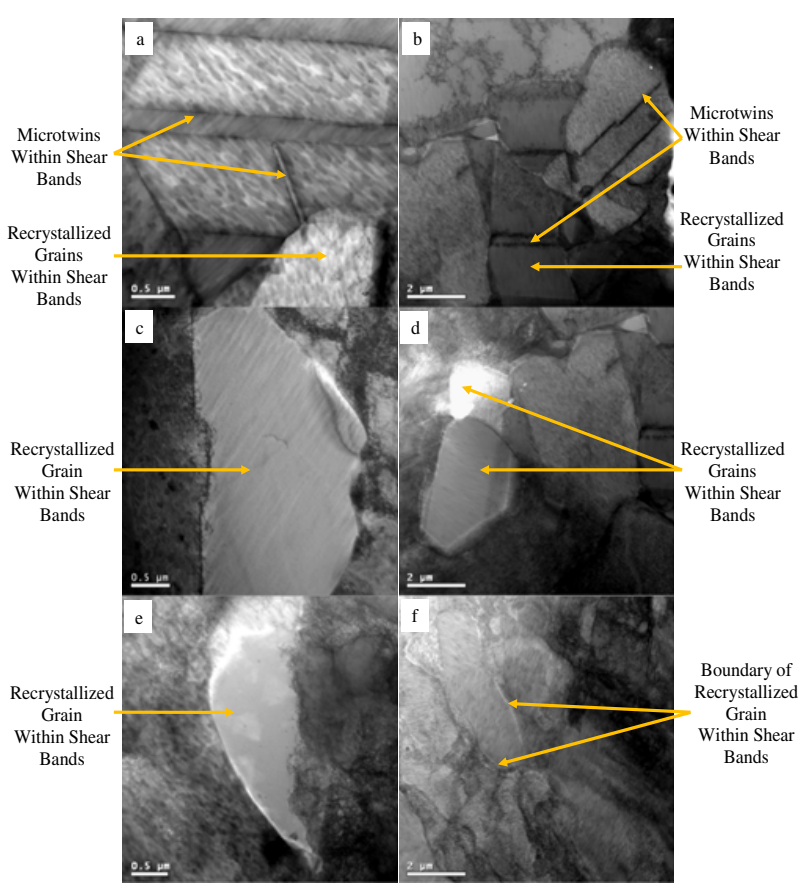

Figure 9. TEM micrographs of regions within an ASB that evolved in a copper specimen after impact at $57 \mathrm{~kg} \cdot \mathrm{m} / \mathrm{s}$ showing evolved recrystallized grains and annealing twins.

dislocations and alignment of the grains in the shear direction (texture development). Elongated grains, broken grains, refined grains, subgrains and nanograins decorated with high density of dislocations were observed within the shear band structures that evolved after the onset of strain localization. However, at every other impact momentum/strain rate, after the onset of strain localization, there were predominant grain structures and microstructural features that characterized the shear band structure. This demonstrate that different mechanisms occur simultaneously including the emergence of dislocations, breaking of grains, and grain refinement during the evolution of the shear bands in the tempered steel specimens depending on the imposed local strain and strain rate.

On the other hand, dislocation multiplication and cell formation, and dynamic recovery and recrystallization characterized the structure within the shear bands that evolved after impact in the copper specimens. There was only a single mechanism that was dominant within the microstructure of the evolved shear band after the onset of strain localization. At relatively lower strain rates, dislocation multiplication and dislocation cell formation characterized the microstructure of the evolved shear bands within the copper specimens. As the impact momentum/strain rate increased, the dislocations and dislocation cells were replaced by recrystallized grains and microtwins. Fine equiaxed grains with low dislocation density and well-defined grain boundaries are the microstructural characteristics of the occurrence of Dynamic Recrystallization (DRX) [1,6]. DRX has been defined as a structural rearrangement as a result of refinement of initial grain structures of materials to a point where new nanograins form and grow [1]. It is inferred that the high impact momentum/strain rate of deformation 
results in the onset of dynamic recrystallization. Prior to the onset of dynamic recovery and recrystallization, the microstructure within the shear band reaches the saturation dislocation density due to dislocation multiplication and dislocation cell formation processes. The excess deformation energy coupled with adiabatic heating raises the temperature of the copper specimens which triggers dynamic recovery and recrystallization processes [7].

\section{Conclusion}

In this study, pre-impact and post-impact microstructure characterization was conducted on tempered 4340 steel (BCC) and pure copper (FCC) specimens to determine the mechanism of evolution of ASBs. This was done in order to determine the effect of crystal structure and microstructure on the mechanisms and appearance of ASBs in both materials. Following the tests, the specimens were examined using optical and electron microscopy. Specimens from both materials exhibited failure by the development of adiabatic shear bands.

The evolution of the shear band structure in the tempered steel specimens can be considered as a simultaneous layering of microstructures initially driven by dislocations which produce the final structures observed in the shear bands at the end of passage of the stress wave. On the other hand, the shear band in the copper specimens evolved by the occurrence of sequential mechanisms including dislocation multiplication and cell formation, and dynamic recovery and recrystallization. The differences in the mechanism of grain refinement and evolution of the shear bands in both materials is attributed to the differences in the mobility of dislocations, the rate of strain hardening and strain hardening exponents in both materials studied.
The financial support of Defense Research and Development Canada (DRDC DND) for this study is greatly appreciated.

\section{References}

[1] B. Dodd, and Y. Bai, Adiabatic Shear Localization: Frontiers and Advances, Elsevier (2012).

[2] Q. Wei, Z. L. Pan, X. L. Wu, B. E. Schuster, L. J. Kecskes And R. Z. Valiev, Microstructure And Mechanical Properties At Different Length Scales And Strain Rates Of Nanocrystalline Tantalum Produced By High-Pressure Torsion, Acta Materialia 59 (2011) 2423-2436.

[3] P. W. Leech, Observations Of Adiabatic Shear Band Formation In 7039 Aluminum Alloy, Metallurgical Transactions A 16 (1985) 1900-1903.

[4] S. Boakye-Yiadom, A. K. Khan and N. Bassim, (2014) A systematic study of grain refinement during impact of 4340 steel. Materials Science and Engineering A 605 (2014) 270-285.

[5] S. Boakye-Yiadom, A. K. Khan and N. Bassim, Effect of pre-impact microstructure on the nucleation and initiation of Adiabatic Shear Bands (ASBs) during impact, Materials Science and Engineering A 615 (2014) 373-394.

[6] Y. Xu, J. Zhang, Y. Bai And M. A. Meyers, Shear Localization In Dynamic Deformation: Microstructural Evolution, Metallurgical And Materials Transactions A 39 (2008) 811-841

[7] A. Mishra, M. Martin, N. N. Thadhani, B. K. Kad, E.A. Kenik, M.A. Meyers, High-strain-rate response of ultra-fine-grained copper, Acta Materialia 56 (2008) 2770-2783. 